\title{
Evolution of MBE HgCdTe defect structure studied with ion milling method
}

\author{
Malgorzata Pociask-Bialy ${ }^{*}$ \\ Faculty of Mathematics and Natural Sciences, University of Rzeszow \\ S.Pigonia 1, 35-959 Rzeszow, Poland
}

\begin{abstract}
In this paper, is shown how ion milling can assist in assessing the defect structure of MCT by revealing the residual doping, and establishing the minimum level of donor concentration $N_{\mathrm{md}}$, which is needed for obtaining n-regions with a reproducible $n$ value. For this purpose, a study of the electrical properties of ion-milled LWIR n-type MCT films, un-doped and doped with indium with the concentration $N_{\text {In }}=5 \times 10^{14}-10^{17} \mathrm{~cm}^{-3}$ is proposed .
\end{abstract}

\section{Introduction}

MCT still is holding its position as the fundamental material for midwave infrared and long- (LWIR) photodetectors. Processing MCT with low-energy ions, about $0,5 \mathrm{keV}$, causes the formation of a source of mercury interstitials $\mathrm{Hg}_{\mathrm{s}}$, which get injected into the bulk of the material due to a very high surface concentration $[1,2]$. Ion milling (IM) propagating into the crystal, the interstitials annihilate the mercury vacancies and react with acceptors to form donor centres. In p-type MCT, this converts a surface layer to n-type and creates a $\mathrm{p}-\mathrm{n}$ junction. In n-type MCT these processes modify the electrical properties of the material [3].

Of the number of techniques used to characterize the quality of MCT, ion milling (IM) is emerging as a unique means to reveal electrically active defects and complexes. It is known that IM is capable of strongly affecting electrical properties of MCT, up to conductivity type conversion in $p$-type material [4]. It appears, that strongly non-equilibrium processes which take place under IM, when material is oversaturated with interstitial mercury atoms $\mathrm{Hg}_{I}$ generated near the surface [1-2], lead to the appearance of specific defect complexes, which may not form under other conditions. These complexes may also comprise intrinsic point and extended defects that normally do not show their presence due to their electrical neutrality or compensation $[4,10]$.

By carefully measuring the parameters of the crystal before and after the milling, and following the disintegration of defects with time after IM ('relaxation'), one can detect and identify these defects.

\section{Experiment}

In this paper we report on applying IM for the study of defects in LWIR MCT films grown by MBE on GaAs substrates with $\mathrm{ZnTe} / \mathrm{CdTe}$ buffer layers. The results obtained on the MBE films grown on GaAs are compared to those acquired on the MBE films grown on $\mathrm{CdZnTe}$ substrates, and on MCT bulk crystals and films grown by isothermal vapor (ISOVPE) phase epitaxy [8].

We have studied nominally un-doped MBE films with composition $x=0.22$ (LWIR) and $x=0.30$ (MWIR), and those in situ doped with an acceptor (As with concentration $10^{15}-10^{16} \mathrm{~cm}^{-3}$ ) or a donor (In with concentration $5 \cdot 10^{14}-10^{17} \mathrm{~cm}^{-3}$ ), both as-grown and after various kinds of post-growth annealing.

The thickness of the MBE films was 7 to $11 \mu \mathrm{m}$, and they were protected by $\sim 0.3 \mu \mathrm{m}$-thick (top) and $\sim 1.0 \mu \mathrm{m}-$ thick (bottom) graded-gap films with $x$ increasing up to $\sim 0.5$ near the both interfaces.

In a typical structure, a $\mathrm{Hg}_{1-x} \mathrm{Cd}_{x} \mathrm{Te}(x=0.22$ for a LWIR structure) 'absorber' film with a thickness $d \sim 8-10 \mu \mathrm{m}$ was protected by $\sim 0.3 \mu \mathrm{m}$-thick (top) and $\sim 1.0 \mu \mathrm{m}-$ thick (bottom) graded-gap films with $x$ increasing up to $\sim 0.5$ near the both interfaces. LWIR films (all investigated samples), both un-doped and in situ Indoped, were grown at a substrate temperature $T_{\text {sub }}$ $\sim 185{ }^{\circ} \mathrm{C}$.

Ion milling was performed using an IB-3 (EIKO, Japan) etching system with $\mathrm{Ar}^{+}$ion energy $500 \mathrm{eV}$, current density $0.2 \mathrm{~mA} / \mathrm{cm}^{2}$ and milling time $10 \mathrm{~min}$. The temperature of the samples during the milling was kept at $\sim 293 \mathrm{~K}$ by means of cooling of the sample holder with water. The relaxation of the electrical properties of

Corresponding author: pociask@ur.edu.pl

C) The Authors, published by EDP Sciences. This is an open access article distributed under the terms of the Creative Commons Attribution License 4.0 (http://creativecommons.org/licenses/by/4.0/). 
the samples was studied by measuring repetitively the Hall coefficient $R_{H}$ and $\sigma$ in magnetic field $B$ of 0.01 up to $1.5 \mathrm{~T}$. The measurements were performed on squareshaped van der Pauw structures. During the measurements the samples were kept at liquid $\mathrm{N}_{2}$ temperature. The $\mathrm{R}_{\mathrm{H}}(\mathrm{B})$ and $\sigma(\mathrm{B})$ dependences were analyzed using Discrete Mobility Spectrum Analysis (DMSA), as described elsewhere [4,9].

First measurements were performed straight after the milling, with the allowance for the time required for mounting samples on the holders and cooling them down to $\mathrm{LN}_{2}$ temperature. This took about 8-15 min for the electrical measurements, and about one hour for the PL experiments. After the measurements, the samples were allowed to warm up to the room temperature, and were stored in air before the next measurement, again performed at the low temperature.

\section{Results and discussion}

Using IM, a unique neutral defect, which forms at the stage of the growth, and which appears to be specific to our MBE films, was revealed.

Straight after IM the electron concentration at $T=77 \mathrm{~K}$ $n_{77}(i)$ in all the MBE films $\left(\sim 10^{17} \mathrm{~cm}^{-3}\right.$ for LWIR films) greatly exceeded that in the bulk and ISOVPE samples (see Fig. 1).

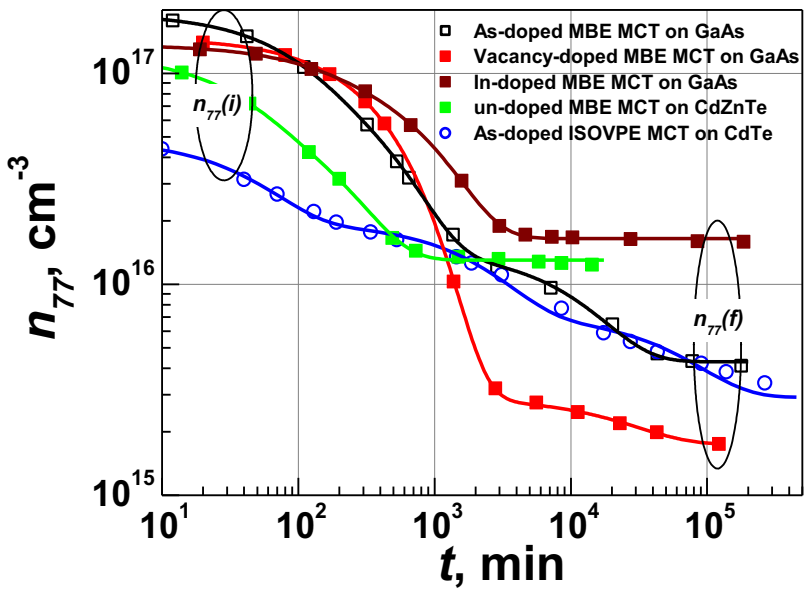

Fig. 1. An example of long-time relaxation of electron concentration after IM in some of the $\mathrm{Hg}_{1-x} \mathrm{Cd}_{x} \mathrm{Te}(x \approx 0.22$ for a LWIR) films studied. Points are experimental data and curves are multi-order exponential fits.

$\square$ As-doped MBE MCT on GaAs, - Vacancy-doped MBE MCT on GaAs, - In-doped MBE MCT on GaAs, a un-doped MBE MCT on CdZnTe, $\circ$ As-doped ISOVPE on CdTe.

The modification front, which forms under the milling, is very sharp, as the $\mathrm{Hg}_{\mathrm{I}}$ migration does not affect the remaining 'core' of the crystal [1]. The properties of the 'core' are determined by the growth or post-growth treatment conditions. As to the modified n-layer, the electron concentration $n$ there is usually determined by the total concentration of stable residual donors $N_{\mathrm{SD}}$ (both native $N_{\mathrm{ID}}$ and extrinsic $N_{\mathrm{ED}}$ ) and the formed donor centres $D$. [5] When the milling stops, the centres gradually disintegrate. As a result of this relaxation process, $n$ decreases, so within $10^{3}-10^{5} \mathrm{~min}$, depending on the chemical nature of acceptors, it stabilizes at the $N_{\text {SD }}$ level [6]. In this way, in a device structure formed by ion milling in nominally un-doped MCT the electrical properties of the $n$-region are defined by residual donors.

For the $n_{77}(i)$ the following expression $[10,11]$ can be write: $n_{77}(i)=N_{S D}+D^{\circ}+N_{N}$

where $N_{N}$ is the concentration of neutral defects, which get electrically activated through the interaction with $\mathrm{Hg}_{\mathrm{I}}$. These defects, as it was underlined earlier, form during the growth and may represent complex native defects, or initially electrically neutral impurity [9]. Cause of presence of the excessive tellurium in the films, possibly in a form of defect clusters [12], these defects may be Te-related.

After the relaxation, with the donor centres and electrically activated defects disintegrated, we have that

$$
n_{77}(f)=N_{S D} .
$$

In the In-doped LWIR films under study: $N_{S D}=N_{I n}+N_{I D}+$ $N_{E D}$ and it is clearly visible on Figure 1 that after $\sim 5 \times 10^{3}$ min of ageing the $n_{77}$ in the In-doped LWIR films (sample $\square$ ) with $N_{I n} \geq 10^{16} \mathrm{~cm}^{-3}$ stabilised at the level of the concentration of the introduced dopant. As we see $n_{77}(f)=N_{\text {In }}$ for the case of sample $\mathbf{m}$. In nominally undoped sample $=$ the $n_{77}$ stabilised at $\sim 2 \times 10^{15} \mathrm{~cm}^{-3}$. For these samples it appears that $N_{I D}+N_{E D}>N_{I n}$ and $n_{77}(f) \approx$ $N_{I D}+N_{E D}$, so the $n_{77}(f)$ reflects the residual donor doping level. Given that the $n_{77}(f) \approx 2 \times 10^{15} \mathrm{~cm}^{-3}$ we can conclude that the as-grown films with $n_{77}(0)<10^{15} \mathrm{~cm}^{-3}$ must been, in fact, compensated. $N_{\mathrm{SD}}$ typically lies in the range $10^{14}$ $10^{15} \mathrm{~cm}^{-3}$ and Its typical for molecular beam epitaxy (MBE) growth technique, conditions, as well as on the chemical purity of the MCT components used. To avoid the uncertainty related to repeatability of $N_{\mathrm{SD}}$, intentional donor doping of the material can be used only for the one presented sample. It is believed that ion milling of donor-doped MCT should result in the fabrication of ntype material, whose electron concentration reflects the doping level $[7,8]$. In reality, however, in low-doped MCT residual donors $\left(N_{\mathrm{SD}}\right)$ may override intentionally introduced dopants [9]. Thus, to control the electron concentration in low-doped $n$-regions formed with ion milling, precise knowledge of the residual doping level is desirable.

The $n_{77}(i)$ in the MBE films depended only on $x$ (i.e., growth conditions), and neither on substrate type, nor on the type and concentration of dopants, nor on the terms of post-growth annealing (Fig. 1).

\section{Conclusions}

Thus, it was concluded that in our MBE films IM led to the formation of a donor center, which resulted from the interaction of $\mathrm{Hg}_{I}$ with a certain defect that had been electrically neutral before the milling. The analysis showed that the most probable candidate was an initially neutral impurity (e.g., oxygen), or a defect complex involving excessive tellurium atoms. The exact nature of 
the defect and its relation to the MBE growth conditions will be discussed in details.

Using IM, we also established donor and acceptor background doping level in the MBE films and made estimates of their electrical compensation. The electron concentration as measured after the relaxation of defects formed under the milling, $n_{77}(f)$, appears to be similar in all the $\mathrm{HgCdTe}$ samples (except for those strongly doped with In), $\sim(1-4) \times 10^{15} \mathrm{~cm}^{-3}$. We relate this feature to the MBE growth conditions that allow for a variety of Terelated defects.

\section{References}

1. V.V. Bogoboyashchyy and I.I. Izhnin, Russ. Phys. J. 43, 627-36 (2000)

2. D. Shaw and P. Capper, J. Mater. Sci.: Mater. Electron. 11, 169-177 (2000)

3. K.D. Mynbaev and V.I. Ivanov-Omskii, Semiconductors 37, 1127-50 (2003)

4. I.I. Izhnin, S.A. Dvoretsky, N.N. Mikhailov, Yu.G. Sidorov, V.S. Varavin, K.D. Mynbaev, and M. Pociask, Appl. Phys. Lett. 91, 132106 (2007)

5. I.I. Izhnin, V.V. Bogoboyashchyy and F. F. Sizov, Proc. SPIE 5881 5881OU1-11 (2005)

6. O. Gravrand, E. De Borniol, S. Bisotto, L. Mollard and G. Destefanis, J. Electron. Mater. 36, 981-987 (2007)

7. K. R. Kurbanov and V.V. Bogoboyashchii, Condens. Media Interface 1, 245-249 (1999)

8. V.S. Varavin, V.V. Vasiliev, S.A. Dvoretsky, N.N. Mikhailov, V.N. Ovsyuk, Yu.G. Sidorov, A.O. Suslyakov, M.V. Yakushev and A.L. Aseev, OptoElectronics Review 11, 99-111 (2003)

9. M. Pociask, I.I. Izhnin, S.A. Dvoretsky, N.N. Mikhailov, Yu.G. Sidorov, V.S. Varavin, K.D. Mynbaev and E. Sheregii, Semicond. Sci. Technol. 23, 095001, 5pp (2008)

10. I.I. Izhnin, S.A. Dvoretsky, K.D. Mynbaev, N.N. Mikhailov, Yu.G. Sidorov, V.S. Varavin, R. Jakiela, M. Pociask, and G. Savitsky, phys. stat. sol. (c) 7, 6 1618-1620 (2010)

11. K.D. Mynbaev, N.L. Bazhenov, M.V. Yakushev, D.V. Marin, V.S. Varavin, Yu.G. Sidorov, S.A. Dvoretsky, Tech. Phys. Lett. 40, 708 (2014)

12. S. N. Yakunin and N. N. Dremova, JETP Letters 87, 494-497 (2008) 